\title{
VOLVIENDO A LAS BASES: EL SINDICALISMO ARGENTINO FRENTE A LOS RETOS DEL CAPITALISMO ACTUAL
}

\section{Bruno Dobrusin ${ }^{\mathrm{a}}$}

aUniversidad de Buenos Aires (UBA), Centro de Estudios e Investigaciones Laborales - CEIL CONICET. Buenos Aires, Argentina. E-mail: <bdobrusin@gmail.com>

Orcid: 0000-0002-0218-1146

\section{Juan Montes Catób}

${ }^{b}$ Doctor en Ciencias Sociales por la Universidad de Buenos Aires (UBA) e Investigador del Centro de Estudios e Investigaciones Laborales (CEIL-CONICET). Co-coordinador del Programa de Estudios Críticos sobre el Movimiento Obrero (PECMO). Docente de la UBA. Argentina, Buenos Aires.

E-mail:<jmontescato@gmail.com>

Orcid: 0000-0002-5835-4878 http://dx.doi.org/10.1590/0102-239258/104

\section{Introducción}

El movimiento sindical argentino se enfrenta a un reto fundamental: continuar con una modalidad de actuación basada en el corporativismo clásico originado en el inicio de los modelos de relaciones laborales a mediados de la década del 1940; o romper con la dependencia en la intervención estatal y retornar a un modelo de acción basado en la presencia en los lugares de trabajo, confrontando directamente con el capital. Este reto no es reciente, pero se acrecienta a partir de la profundización de la globalización neoliberal que agranda el poder del capital en relación al Estado y a los trabajadores (Harvey, 2005). La extensa tradición de lucha sindical en Argentina se compone de momentos históricos donde la 
confrontación directa, en un marco de represión e ilegalidad, tuvo predominio a comienzos del siglo XX y durante las diferentes dictaduras militares; y momentos de mayor legalidad, con activa intervención del aparato estatal como mediador frente a los conflictos con el capital. Esta última forma, con características corporativistas clásicas, dominó el escenario político-sindical desde el surgimiento del peronismo en adelante.

El proceso de cambio se comienza a expresar con claridad hacia los años 90, luego de una década del 80 con activa militancia sindical, cuando las políticas neoliberales logran mermar el poder sindical, disminuir la afiliación y la capacidad de organizarse en los lugares de trabajo, histórico bastión del movimiento obrero (Montes Cató, 2004; Etchemendy, 2011). El debilitamiento estructural no fue total. El sindicalismo entró en problemas, pero no necesariamente en crisis terminal. Las estructuras sindicales se reciclaron hacia otros fines (provisión de servicios), manteniendo la incidencia política en el 240 gobierno y en la oposición (Murillo, 2005).

Este artículo argumenta que los cambios económicos estructurales producidos a partir de la profundización del neoliberalismo, de la concentración y extranjerización empresaria (Wainer, Gaggero y Schorr, 2014), hirieron al movimiento sindical en el núcleo duro de su organización. Esta herida produjo una caída del poder estructural del sindicalismo en relación al capital y al Estado, expresada, entre otras cuestiones, en la merma de la tasa de densidad sindical por debajo del 40\% (Roberts, 2007). La recuperación económica experimentada durante los años post-crisis 2001, en particular durante los gobiernos neodesarrollistas del kirchnerismo, lograron una revitalización temporaria del poder sindical, sin consolidar ese "retorno del gigante" (Etchemendy, 2011) que se esperaba con mayor incidencia económica y política.

La dependencia del sindicalismo sobre métodos basados en la etapa corporativista (con un Estado activo, una economía semi-cerrada y un fuerte componente de capital privado 
de corte nacional) declinó el poder sindical en la actualidad, ya que estas prácticas son insuficientes para superar las limitaciones estructurales políticas y económicas sobre el sindicalismo y sobre el propio Estado. Volver a las bases significa, en palabras de Poulantzas, desbordar los espacios estatales (Poulantzas, 1980), retomando un período de mayor confrontación directa con el capital. Este, entendemos, es el principal desafío del movimiento sindical argentino en la etapa actual.

El trabajo está divido en cinco apartados. El primero desarrolla los componentes principales del modelo corporativista. El segundo se centra en los cambios y transformaciones recientes del mundo laboral. El tercero aporta un análisis sobre nuevas formas de conflictividad. El cuarto retoma las líneas históricas respecto a la representación y organización en los lugares de trabajo. La última sección introduce las conclusiones.

\section{Lógicas de acción en el sindicalismo argentino}

En Argentina, la CGT tiene una presencia política extendida en el tiempo, con incidencia sobre la política nacional desde antes de su creación -y unificación- en 1930. Los gobiernos peronistas se nutrieron del accionar de la CGT, que ya tenía un recorrido histórico importante y de hecho permitió consolidar al movimiento liderado por Perón. La CGT y el peronismo, más allá de la alianza histórica que mantienen, nunca tuvieron una relación de afinidad total, y sí se puede caracterizar como una relación caracterizada como de tensión entre ambos sujetos políticos (Murmis y Portantiero, 2004; Torre, 2004, 2012). Tanto durante los gobiernos peronistas, cuando la conformación del Partido Laborista era la opción promovida por sectores dentro de la central sindical, como en los procesos donde el peronismo estuvo proscripto, nunca se produjo un acuerdo total en torno a cómo entablar la relación entre estos dos actores (James, 1988). La integración y la resistencia, tanto en la época de proscripción como después, son características distintivas de la dinámica entre la CGT y el 
peronismo. En términos interpretativos generales, esta configuración de las formaciones sindicales argentinas responde a lo que se ha dado llamar como organización corporativista.

El corporativismo ${ }^{1}$ implica una relación de intermediación entre el Estado y los dos grupos organizados más relevantes, el empresariado y los trabajadores, cuyas organizaciones obtienen monopolio de reconocimiento y, en la mayor parte de los casos, financiamiento por parte del Estado (Kay, 2003). En sus orígenes, los modelos corporativos funcionaron como una forma de contención del conflicto social, en especial en los países donde el activismo sindical mayoritario tenía características rupturistas respecto al régimen económico y político. Argentina, Brasil y Uruguay son casos paradigmáticos de esta situación, aunque se encuentran también condiciones similares en otros países de la región (Zapata, 1993). A los fines de la argumentación de este trabajo, se refuerza la idea que, en los modelos corporativos, el conflicto intentaba ser contenido dentro de los márgenes del 242 Estado y tenía al aparato estatal como principal objetivo.

El espacio estatal es comprendido a partir de los trabajos de Nicos Poulantzas (1980, 2012), en especial su propuesta respecto a que los espacios materiales e inmateriales del Estado representan un régimen de condensación de la lucha de clases. Para Poulantzas, existen dos concepciones del Estado que imposibilitan contar con una comprensión acabada de la dinámica social. Por un lado, el Estado como cosa supondría que este es simplemente un instrumento manipulable por una sola clase o fracción, y que no posee ninguna autonomía. La segunda interpretación, supone al Estado como sujeto. Aquí la autonomía sería absoluta, porque el poder es concentrado por un Estado con racionalidad propia. Frente a estas concepciones, el autor propone comprender al Estado como una relación, y más exactamente como la condensación material de

\footnotetext{
${ }^{1}$ Se recuperan en este apartado algunas nociones expuestas de manera más amplia en Dobrusin y Montes Cató (2017).
} 
una relación de fuerzas entre clases y fracciones de clase, que se expresa en el seno del Estado. Esta definición evita tomar el Estado solo como instrumento de las clases dominantes o como sujeto aparte del resto de los sujetos políticos, concepción esta última que se encuentra en la base de la corriente institucionalista-funcionalista, predominante en los análisis sobre el sindicalismo en la región. Esta segunda visión está en parte basada en una confusión entre el Estado como espacio y lo que se refiere al aparato estatal. La visión funcionalista refuerza la idea que la burocracia estatal - el aparato del Estado- opera de manera autónoma respecto a los actores de la sociedad y a las disputas de la lucha de clases.

En esta perspectiva, la conflictividad sindical tiene razón de ser en su orientación hacia el espacio estatal, ya que en éste se ven reflejadas también las posiciones de las fracciones hegemónicas del capital. El avance más importante de los movimientos sindicales en conquistas de derechos y en participación en las decisiones relevantes de política económica, se produjo a partir de la integración en los espacios estatales. Lo que Berins Collier y Collier (1991) denominan como 'procesos de incorporación', se pueden ver también como conquistas logradas por el movimiento sindical a partir de la lucha de clases (Murmis y Portantiero, 2004). En definitiva, la trayectoria sindical de acción conflictiva en el marco de los espacios estatales dio sus frutos a partir de la mejora relativa de la situación del movimiento obrero durante los procesos desarrollistas (Nosiglia, 1983). Recuperando la tradición de los estudios que interpretan lo político también con una construcción desde abajo y una mirada activa de los sujetos sociales, entendemos que existen varios argumentos que rebaten ciertas lecturas lineales y empobrecedoras de la política, que reduce su accionar a la planificación premeditada y calculada del gobierno en pos de sus intereses instrumentales.

El accionar sindical al interior del espacio estatal no significa que las luchas directas, de corte clasistas, entre capital y 
trabajo estaban ausentes, pero la preponderancia fue, en la trayectoria del corporativismo, dada por la centralidad del espacio y del actor estatal. Esta particularidad estuvo especialmente presente en el caso de las cúpulas sindicales que, a excepción de aquellos países donde continuaron las férreas relaciones con los partidos clasistas, perdieron el eje del combate hacia el capital y se orientaron hacia la negociación con y en el Estado. Esto tenía que ver con las mejoras objetivas obtenidas por la clase trabajadora y también por la dirigencia sindical durante los períodos de gobiernos que promovieron el corporativismo (Berins Collier y Collier, 1991).

El avance del neoliberalismo en los años 1970 fue cambiando la relación de fuerzas entre el capital y el trabajo. El neoliberalismo no fue solo un discurso ni una teoría sobre las libertades individuales y el libre mercado, sino un proyecto de poder en beneficio de los grandes capitales monopólicos (Harvey, 2007; Crouch, 2011). Esta situación alteró la capaci244 dad del sindicalismo de incidir sobre las políticas económicas y de desarrollo, que pasaron a estar sujetas a las prioridades de las EMN. El crecimiento exponencial fomentado por la concentración de mercados y la extranjerización de las economías dejó al sindicalismo frente una situación compleja, y con la necesidad de reacomodarse ante el nuevo panorama. Las luchas de la post-democratización por derechos sociales, económicos y políticos (Valenzuela, 1989) movilizaron al actor sindical convirtiendo a las luchas por ciudadanía en luchas económicas. Sin embargo, esas luchas no se tradujeron en una alteración significativa de los esquemas productivos, que ya comenzaban a dar señales del avance del capital transnacional y de una caída en la participación del sindicalismo en las decisiones económicas.

Durante los años 1990, la caída de la conflictividad clásica, medida en huelgas y horas paradas por los trabajadores, fue clave para el avance del proceso neoliberal, que profundizó la crisis de estas organizaciones. El conflicto sindical 
fue mutando hacia esquemas de movilización basados en la ocupación de los territorios, en desmedro del eje sobre los espacios productivos.

\section{Transformaciones laborales y desafíos al accionar tradicional}

El corporativismo como modelo de accionar sindical enfrenta límites impuestos esencialmente por los cambios en los modelos de acumulación del capital. Las mejoras socioeconómicas de los mandatos del kirchnerismo en Argentina no lograron consolidar un nuevo sistema de relaciones Estado, sindicatos y empresarios. La concentración y extranjerización del capital se profundizó durante la etapa, siguiendo la línea trazada a comienzos de los años 1990 (Wainer, Gaggero y Schorr, 2014). La mayor presencia estatal mediante el incremento de los impuestos al campo, la ampliación de los beneficios sociales y el crecimiento de la negociación colectiva (Dobrusin y Campos, 2015) no consolidaron un régimen de acumulación alternativo. Los gobiernos neodesarrollistas reforzaron la presencia del Estado en áreas claves de la economía, produjeron procesos redistributivos con eje en el mercado interno, y al mismo tiempo mantuvieron una estructura productiva y de exportación que consolidó el modelo implantado con el neoliberalismo. La dependencia en las estrategias de cadenas productivas de empresas multinacionales es un factor que no logró ser revertido durante los neodesarrollismos, lo que distingue del proceso desarrollista clásico, cuando las empresas estatales tenían preponderancia en los sectores más importantes de la economía.

Los cambios en el mercado de trabajo hacia la predominante precarización laboral y la informalidad como un dato de la realidad del mercado de trabajo se mantuvieron durante los gobiernos neodesarrollistas. La informalidad descendió de manera constante a partir de 2003, produciendo uno de los descensos más importantes en la región (Betranou y Casanova, 
2015), pero a 2010 todavía se mantenía en niveles que superaban el $40 \%$ del total de trabajadores ocupados (Ibídem en base a datos de OIT). El empleo asalariado no registrado tuvo la baja más expresiva, del 49\% en 2003 a un 34,6\% en 2012 (Betranou y Casanova, 2015, p. 38). Ese núcleo duro de informalidad se comenzó a expandir nuevamente a partir de 2014 (OIT, 2016), siendo ya una característica de la economía argentina de los últimos 30 años. La representación sindical de trabajadores informales es una de las problemáticas que debe enfrentar el sindicalismo en la etapa actual. Una segunda es la organización contra la precariedad laboral que se extiende inclusive dentro del sector empleado formalmente (OIT, 2016). Comienzan en Argentina a surgir nuevas organizaciones sociales que apuntan a organizar a ese conjunto de trabajadores, en especial la Confederación de Trabajadores de la Economía Popular, pero lo hacen en paralelo a las principales organizaciones sindicales.

La persistencia de acuerdos de libre comercio, acuer246 dos Bilaterales de inversión, y los acuerdos marco de la Organización Internacional del Comercio, crean grandes restricciones al accionar del Estado como regulador y actor en la economía, pero también por sobre los sindicatos como organizadores en los lugares de trabajo. La histórica dependencia en la presencial del Estado como mediador es insuficiente para confrontar a un capital internacionalizado y cada vez más concentrado. En un artículo reciente, la académica norteamericana Ruth Milkman (2013) proponía que los sindicatos tenían que "volver al futuro". El argumento de Milkman es que el crecimiento original de los sindicatos se dio a comienzos del siglo XX, con la intervención del Estado a favor de los empresarios, en un ambiente hostil, de concentración económica y de amplias desigualdades. El accionar sindical se basó en la presencia en los lugares de trabajo, la utilización de la huelga (inclusive de forma ilegal), y la construcción de lazos transnacionales (Milkman, 2013, p. 648). Esta descripción puede ser extrapolada al contexto argentino de comienzos del siglo XX, 
con el auge del sindicalismo anarquista, comunista y socialista. La situación actual, con un nuevo gobierno de corte empresarial, empuja a los sindicatos a rever sus estrategias en base a un contexto diferente de aquel en el cual se produjo un auge sindical. El corporativismo tuvo un rol fundamental en defender conquistas durante los años 1990, pero es insuficiente para el panorama político, económico y social actual. Siguiendo la propuesta de Milkman, el sindicalismo argentino tiene en su propia historia, y en sus propias prácticas de lucha, una referencia para actuar en la coyuntura del neoliberalismo recargado que se vive en la actualidad.

\section{Rasgos de la conflictividad: nuevas relaciones y sujetos colectivos}

Puede señalarse que, en cuanto al protagonista de la conflictividad, exceptuando el período de agudización de la crisis durante el 2001, el actor sindical tuvo un despliegue significativo. La irrupción de las organizaciones de desocupados tuvieron sin embargo un impacto fuerte en vista del cambio en la lógica de representación, ya que estas últimas poseen un arraigo territorial sumamente importante (en esta misma tendencia, las organizaciones de los trabajadores de la economía popular en la actualidad se emparentan con las organizaciones de desocupados). Ahora bien, si la novedad en términos de luchas sociales vino de la mano de las organizaciones de desocupados, ello es parte de un fenómeno más amplio de luchas defensivas que se revierte a partir del 2003, período donde comienza a revertirse este fenómeno de la mano de un aumento de la conflictividad ofensiva demandando mejoras en los salarios y condiciones de trabajo, aunque la primera de estas demandas se encuentre atada al poder adquisitivo del salario real por el proceso inflacionario.

A partir del año 2003 los conflictos laborales estuvieron orientados, en términos generales a la recuperación del salario real, que había caído a los mínimos históricos en el año 2002. 
Este proceso se refleja en el contenido de la materia negociada como en los motivos de la conflictividad. En este marco, se destacó una participación muy significativa de los niveles centralizados de la estructura sindical (federaciones y uniones), y por ello mismo de los conflictos por rama de actividad. En el año 2008 tiende a modificarse esta tendencia cuando diversos indicadores comenzaron a dar cuenta de una creciente descentralización de los conflictos (ODS, 2011).

Si bien el fenómeno al que hacemos referencia es difícil de mensurar, puede ser caracterizado a través de ciertos indicadores que permiten aproximarnos al tema en términos cuantitativos. Por un lado, se verifica una amplia cantidad de conflictos circunscriptos al ámbito de la empresa, que representan el $78 \%$ del total en desmedro de los conflictos a nivel de actividad o rama (ODS, 2010). En segundo lugar, se consolida un crecimiento de los reclamos promovidos por sindicatos locales o seccionales, que explican el $64 \%$ de los casos 248 en el 2009 (frente a un 51\% en el año anterior). En tercer lugar, persiste una importante cantidad de conflictos (un 14\% del total) llevados adelante en espacios de trabajo en los que no se consigna la presencia de representantes sindicales, o se trata de colectivos que poseen una representación de hecho (muchas veces opuesta a la conducción de la organización sindical) construida en el desarrollo del propio conflicto. Esto último es significativo, porque señala la existencia de conflictos que son encabezados por activistas que actúan como delegados "de hecho".

A partir del 2009, también se ha profundizado una tendencia hacia la descentralización de los conflictos laborales, tanto en lo que hace a los sujetos que participan en ellos, como al ámbito en el que se producen. A partir de entonces, comenzó a crecer la participación de los sindicatos de base y de las secciones en los conflictos. Ellos pasan de representar el $50 \%$ del total en los años 2007 y 2008, a alrededor del $65 \%$ en los años 2009 y 2010, y llevan a un $78 \%$ en el año 2011. Como 
contrapartida, se registra una retracción de la intervención de las federaciones y de las uniones, que pasan de explicar más de un tercio de los conflictos laborales en 2007 y 2008, a poco menos de un $10 \%$ en el año 2011.

Otro dato importante es la canalización de conflictos por fuera de las instituciones gremiales, dando cuenta de la inexistencia de organizaciones sindicales o de las contradicciones entre las bases y dirigencias. Esta observación se complementa con el análisis del ámbito de los conflictos. En el sector privado, los conflictos por empresa pasan de representar el $68 \%$ en 2007 al $82 \%$ en 2011, con un movimiento inverso de los conflictos por rama de actividad.

Este auge de conflictos en los espacios productivos (en las empresas en lugar del nivel de la actividad como aquellos motorizados exclusivamente por comisiones internas) constituye una novedad en el campo sindical en vista de la recuperación de una tradición obrera de lucha situada en el lugar de trabajo que permitió la consolidación y desarrollo de nuevas experiencias sindicales de base de la mano de una repolitización de la militancia gremial.

\section{La emergencia del lugar de trabajo para confrontar al capital}

El contexto sociopolítico post-crisis 2001 implicó, para el movimiento sindical, enfrentar múltiples desafíos profundizados en el período anterior, donde el capital logró asumir la iniciativa política imponiendo una agenda de discusión exclusivamente asociada a sus intereses y encorsetando la intervención sindical a instancias institucionales por fuera de los espacios productivos. La ofensiva sobre los sindicatos adquirió una intensidad inusitada. Sus efectos son conocidos: supuso la destrucción de puestos de trabajo y la precarización de las relaciones laborales, el deterioro de las condiciones de trabajo, la erosión de un conjunto de normas protectoras, la implementación de rígidas normas disciplinarias 
(simultáneamente a nuevas políticas manageriales de implicación desdibujando de ese modo los contornos de intervención de los delegados) y el deterioro del poder sindical. Todas ellas articuladas fueron devastadoras para la intervención sindical en los lugares de trabajo.

Probablemente, la despolitización de los espacios de trabajo -entendida como ausencia de organización colectiva de los trabajadores- sea una de las marcas más potentes que permite articular estratégicamente los efectos del discurso presuntamente desideologizado del neoliberalismo y de la post-dictadura. Esto se vio reforzado por un proceso de desmovilización promovido por algunos dirigentes sindicales (en especial por aquellos que condujeron la CGT durante los noventa) que asumieron esta estrategia a cambio de la acumulación de recursos políticos principalmente orientados a transformar sus organizaciones en sindicatos de corte empresarial.

A pesar de la enorme fuerza puesta a disposición de estos 250 objetivos, en plena hegemonía neoliberal-privatizadora emergieron algunas experiencias de recomposición política en el interior de los espacios de trabajo que marcaron procedimientos, dinámicas de relacionamiento y desafíos que guiaron las futuras luchas obreras. En gran medida, algunos de los rasgos distintivos de estas experiencias recuperaban elementos históricos de la experiencia sindical de base: militancia, politización de los espacios productivos, modelo asambleario y discurso opositor, y se articulaban con un conjunto heterogéneo de luchas populares instaurando nuevos repertorios y simbologías vinculadas a la conflictividad social, re-actualizando de ese modo las tradiciones de lucha.

Muchas de las expresiones de resistencia organizada en los lugares de trabajo fueron de la mano de las comisiones internas en sectores que habían sufrido el vendaval privatizador y de ajuste (estatales, telefónicos, ferroviarios, petróleo, subtes), que encontrarán un contexto más propicio para la expresión pública de la resistencia en el marco de la crisis institucional 
del gobierno en el 2001, y sentarán las bases para que en la siguiente década vuelva a desarrollarse con intensidad la experiencia de las comisiones de fábrica.

La presencia de estas organizaciones en los centros productivos tensiona dos relaciones que resultan importantes cuando de lo que se trata es de comprender el conjunto de vínculos en los que interviene el sindicalismo. Por un lado, la relación con el capital, en la medida que constituye una forma de poder que disputa el control del proceso de trabajo y las condiciones de extracción del plusvalía; por otro, con las esferas sindicales, en cuanto colocan el tema de la autonomía en el centro de debate. Esta tensión se configura a partir del carácter dual que constituye a las organizaciones sindicales de base que, por un lado, despliegan cierta capacidad de disputar el control de la producción y, por lo tanto, la rentabilidad empresarial, y, por otro lado, se colocan en un lugar neurálgico en tanto desafían el control de la burocracia sindical sobre los trabajadores.

Un factor de gran importancia que operó alentando estas transformaciones fue el avance jurisprudencial en materia de protección de la actividad sindical en general, y de la libertad sindical en el espacio de trabajo en particular, que contribuyó a reafirmar la capacidad de acción de los niveles de base. En este plano, el pronunciamiento de la Suprema Corte de Justicia de la Nación en un caso testigo fue el mayor gesto de respaldo institucional y claro indicador de que los extendidos cuestionamientos al modelo sindical basado en el monopolio de la representación empezaban a tener una traducción institucional de alto nivel con serias consecuencias políticas en el mediano plazo $^{2}$.

2 En el fallo "Asociación Trabajadores del Estado clM de Trabajo" S.C.A, 201, L. XL. del 11 de noviembre de 2008, la Corte establece un fuerte cuestionamiento a una cláusula del artículo 41 de la Ley de Asociaciones Sindicales que dispone que, para ejercer las funciones de delegado del personal o miembro de comisiones internas u organismos, "se requiere: a) Estar afiliado a la respectiva asociación sindical con personería gremial y ser elegido en comicios convocados por esta”, considerándolo violatorio 
El fallo dispone que en los lugares de trabajo pueden elegirse delegados que no pertenezcan a la asociación sindical con personería gremial. El pronunciamiento de estas sentencias, que reconocía numerosos antecedentes de tribunales de menor alcance, tuvo consecuencias muy concretas, tanto en los procesos ya en curso - con la reinstalación de sindicatos en formación o de simple inscripción-, como para las experiencias incipientes, en la medida que se constituyó como una garantía de amparo legal a la libre iniciativa gremial en los espacios de trabajo. Además, en cierto sentido, también es posible pensar que el surgimiento de estas dos sentencias, que cuestionan tan severamente ciertos pilares del modelo sindical, constituyen una cristalización institucional -de máximo nivel- de un proceso subyacente, que señalábamos anteriormente, de cierta recomposición de la representación directa de los trabajadores en los lugares de trabajo, que en alguna medida entró en tensión con la normativa establecida e instaló un conflicto que fue canalizado institucionalmente, en este caso, por vía judicial.

En términos de la ubicación de estos procesos de organización y lucha de mínima escala en sectores productivos o casos concretos, aunque no hay información rigurosa sistematizada, en principio es posible decir que estas experiencias aparecen en distintas zonas geográficas y en un amplio espectro de actividades ${ }^{3}$.

Vale destacar que estas experiencias constituyen un conjunto sumamente heterogéneo, tanto en lo que refiere a las características de sus formas de organización y sus conflictos,

del derecho de asociación sindical previsto en el artículo 14 bis de la Constitución Nacional y en diversos tratados internacionales suscriptos por la Argentina.

3 Subterráneo de Buenos Aires, línea 60 de colectivos, ferroviarios del ramal Mitre, choferes de UOCRA), telecomunicaciones (call centers), comercio (supermercados, como Wall Mart), industria química (Praxair), metalúrgica (EMFER, CAT, ASIMRA, Paraná Metal), alimentación (Terrabusi, Pepsico, Stani, Kraft Foods, Wertheim, Sindicato de la Carne), transportistas (Iveco, SIMECA), Prensa (diario Perfil, Radio Nacional, diario La Jornada de Chubut, diario Clarín, diario Popular, TELAM, Editorial Colihue), textil (fábrica Spring, Mafias, tinturerías Pagoda) y bancarios (banco Credicop). 
como a los objetivos políticos que se plantean a partir de la conformación del colectivo de base. Así, encontramos experiencias que se plantean un horizonte político de proyección e institucionalización a partir de la conformación de sindicatos paralelos a los establecidos (el caso paradigmático es el del subte), otras en las cuales se propone un crecimiento político al interior de la estructura sindical propia, y otras que o bien no pretenden o no pueden sostener un crecimiento político más allá del nucleamiento en el espacio de trabajo o de los conflictos puntuales, por lo cual se formalizan y persisten como comisiones internas y cuerpos de delegados, o se desarticulan una vez resueltas - para bien o mal- las reivindicaciones específicas. Puede sostenerse que, entre otros factores, el tipo de relación política entablada con la estructura sindical "oficial”, y la posibilidad de articulación con otras experiencias (dentro y fuera del ámbito estrictamente sindical) es un elemento clave para definir la trayectoria y las posibilidades de estas organizaciones primarias.

\section{Conclusiones}

La ofensiva neoconservadora que se despliega en varios países Latinoamericanos, luego de una etapa caracterizada por políticas neodesarrollistas y ampliación de derechos laborales de principios del siglo XXI, busca erosionar la capacidad de resistencia del movimiento obrero para imponer todo un repertorio orientado a ampliar la informalidad del mercado de trabajo y profundizar la flexibilidad de las condiciones laborales. Desarticular la organización y lucha de los sindicatos se vuelve una etapa clave de la restauración neoliberal.

Para comprender este proceso, hemos destacado que uno de los grandes desafíos para las formaciones sindicales es el de desmarcarse de las limitaciones que lo constriñen seguir insistiendo en la lógica neocorporativa. De este modo, el sindicalismo en Argentina ha estado históricamente caracterizado por regirse bajo sistemas de tipo 'corporativistas', tal como lo 
han definido diversos autores (Berins Collier y Collier, 1991; Murillo, 2005; Cook, 2007; Fernández, 2007; Berins Collier y Handlin, 2009; Etchemendy, 2011). El rasgo principal de este sistema de relaciones está dado por la centralidad del Estado como espacio, institución, de participación y negociación de los actores sociales claves: empresarios y sindicatos. Esta dinámica tripartita caracterizó el desarrollo del accionar sindical en Argentina desde el auge del peronismo en adelante. Los procesos de industrialización por sustitución de importaciones -ISIque se produjeron en la segunda posguerra y hasta mediados de los años 1970 profundizaron el accionar sindical corporativista. La principal característica de estos procesos fue una economía semi-cerrada que priorizaba actores como el empresariado nacional en industrias pesadas y el sindicalismo, dando a cada uno de estos actores roles monopólicos en sus respectivos 254 espacios de incidencia. La centralidad del actor estatal y la permanencia del corporativismo como sistema de ordenamiento de actores empresariales y sindicales es un rasgo destacado de las relaciones del trabajo en Argentina, especialmente si se consideran los cambios rupturistas entre los diferentes procesos económicos, del desarrollismo del ISI al neoliberalismo de las décadas de 1980 y 1990, y de este al neodesarrollismo de los gobiernos en la primera década de 2000.

Como indica Kay (2003, p. 291), el sistema corporativo no solo resistió los cambios en las políticas económicas de la región, sino que también en diversos casos -Brasil, México, Argentina-fue un factor esencial para llevar adelante esos cambios. La incidencia de los actores corporativos es continua. La capacidad de ordenar las relaciones capital-trabajo, inclusive en contextos de debilidad del actor sindical, es una cualidad distintiva de Argentina que perdura en el tiempo (Cook y Bazler, 
2013), permitiendo a su vez que los actores de ese tripartismo se mantengan como protagonistas ${ }^{4}$.

Los autores que se han enfocado en el sindicalismo comparado en América Latina han puesto el enfoque central en las acciones del Estado como factor determinante del accionar sindical. Esto tiene esencialmente que ver con el modelo de relaciones laborales y con la historia de la mayoría de los países, donde el corporativismo instalado como forma de mediación entre sectores divergentes se construyó a partir del Estado. Así, el trabajo de Berins Collier y Collier (1991) toma lo que determinan "coyunturas críticas" que permitieron cambios sustanciales en las relaciones de los Estados con los movimientos sindicales de la región. Estas coyunturas son momentos de crisis y transformación política, de un régimen a otro, que permiten formatear la realidad de una manera decisiva para los tiempos subsiguientes. En estas coyunturas, suelen dominar los clivajes entre los trabajadores y el Estado, así como los trabajadores y los empresarios. Los procesos de incorporación por parte del Estado responden a esas coyunturas críticas, y permiten, a través de esa participación en instancias de negociación, el control de los conflictos para el Estado, así como también para las organizaciones una canalización de las demandas. El trabajo de Berins Collier y Collier no niega que hayan existido alternativas a este modelo centralista en América Latina, en especial a través de movimientos anarquistas y autonomistas, pero refuerzan que la dinámica costo-beneficio era ampliamente a favor de integrarse a los modelos corporativistas. Argentina cuenta con una trayectoria importante de sindicalismo anarquista, pero fue bajo el paraguas corporativista que se expandió el accionar

\footnotetext{
${ }^{4}$ Este protagonismo es puesto cuestión en base a los análisis sobre los límites de los procesos redistributivos que promovió el kirchnerismo. Berins Collier y Etchemendy (2007) ya comienzan a marcar diferencias al hablar de un "neocorporativismo segmentado", que no tiene el mismo alcance que en épocas de ISI. El crecimiento de la informalidad sumado a la precariedad laboral y al poder transnacional de las empresas colocan en cuestión el protagonismo sindical y el corporativismo como sistema. Este debate es profundizado en el capítulo 5 y luego retomado en la conclusión.
} 
sindical y que consolidó a la CGT como un actor significativo en el contexto político-económico argentino.

El auge neoliberal de los años 1990 comenzó a minar la capacidad de acción sindical clásica de los modelos corporativistas. La privatización de empresas estatales, combinada con la flexibilización de los contratos de trabajo y el avance de las empresas multinacionales por sobre los propios estados, puso al corporativismo en jaque. Más allá de una breve recuperación de la capacidad de incidencia sindical durante los gobiernos neodesarrollistas entre 2003 y 2015, la debilidad estructural del actor sindical perdura. Ese corporativismo eficiente de una economía semi-cerrada es insuficiente para enfrentar los retos actuales que viven los trabajadores. Desbordar los espacios estatales no significa una estrategia independiente de lo que ocurra en el Estado, sino una complementación de la participación en los espacios estatales con una estrategia de confrontación y disputa directa hacia el capital, en especial en su versión transnacional. Entre los principales elementos para llevar adelante esa estrategia, se torna necesario rediscutir las relaciones de producción (Mello y Silva, 2016), y reformular los proyectos sindicales en vistas de las limitaciones del Estado en la coyuntura actual.

\section{Bruno Dobrusin}

es doctor en Ciencias Sociales por la Universidad de Buenos Aires (UBA) y becario de posgrado del Centro de Estudios e Investigaciones Laborales (CEIL-CONICET). Docente del Global Labour University.

\section{Juan Montes Cató}

es doctor en Ciencias Sociales por la Universidad de Buenos Aires (UBA) e Investigador del Centro de Estudios e Investigaciones Laborales (CEIL-CONICET). Co-coordinador del Programa de Estudios Críticos sobre el Movimiento Obrero (PECMO). Docente de la UBA. 


\section{Bibliografía}

BERINS COLLIER, Ruth; COLLIER, David. 1991. Shaping the Political Arena: critical conjunctures, the labor movement and regime dynamics in Latin America. Princeton: Princeton University Press.

BERINS COLLIER, Ruth; ETCHEMENDY, Sebastián. 2007. Down but Not Out: Union resurgence and segmented neocorporatism in Argentina (2003-2007). Politics E Society, v. 35, n. 3, pp. 363-401.

BETRANOU, Fabio; CASANOVA, Luis. 2015. Las instituciones laborales y el desempeño del mercado de trabajo en Argentina. Buenos Aires: OIT.

COOK, Maria Lorena. 2007. The politics of labor reform in Latin America: between flexibility and rights. University Park: The Pennsylvania State University Press. 2011. Unions and Labor Policy under left governments in Latin America. Revista Trabajo, v. 5, n. 7, pp. 55-73.

COOK, Maria Lorena; BAZLER, Jonathan. 2013. Bringing Unions back in: labour and left governments in Latin America. Cornell ILR Working Paper, n. 166, pp.1-45.

DOBRUSIN, Bruno; CAMPOS, Luis. 2016. Labour conflict in Argentina and Brazil: challenging an alliance?" Workers of the World: International Journal of Strikes and Social Conflict, v. 1, n .8, pp. 99-121.

DOBRUSIN, Bruno; MONTES CATÓ, Juan. 2017. Repensar el conflicto sindical latinoamericano frente a las estrategias del capital transnacional. Revista Theomai, n. 36, pp. 1-17.

ETCHEMENDY, Sebastián. 2011. Models of economic liberalization: business, workers and compensation in Latin America, Spain and Portugal. Cambridge: Cambridge University Press.

FERNÁNDEZ, Arturo. 2007. Estado y sindicatos en perspectiva latinoamericana. Buenos Aires: Prometeo Libros.

HARVEY, David. 2007. Breve historia del neoliberalismo. Madrid: Akal. KAY, Paul. 2003. Trying on the Emperor's New Clothes? Concertación, Corporatism and Neoliberal Restructuring in the Semiperiphery. Bulletin of Latin American Research, v. 22, n. 3, pp. 291-318.

MELLO E SILVA, Leonardo. 2016. Inovaçoes do sindicalismo brasileiro em tempos de globalização e o trabalho sob tensão. In: SINGER, Andre; LOUREIRO, Isabel. (eds.). As contradiçoes do lulismo: a que ponto chegamos? São Paulo: Boitempo. pp. 93-121.

MILKMAN, Ruth. 2013. Back to the future? US labor in the New Gilded Age. British Journal of Industrial Relations, v. 51, n. 4, pp. 645-665.

MONTES CATÓ, Juan. 2014. Los comités de fábrica en Brasil y Argentina: trayectoria y recomposición del sindicalismo de base. Revista Latinoamericana de Estudios del Trabajo, n. 31, pp. 229-254. 
MINISTERIO DE TRABALHO, EMPLEO Y SEGURIDAD SOCIAL. 2010. Informes especiales sobre conflictos laborales 2006-2010. Asunción: MTESS. Disponible en: <http://www.trabajo.gov.ar/left/estadisticas/negcol/ informesa.asp >. Acceso en: 24 feb. 2018. 2011. Informes especiales sobre conflictos laborales 2006-2010. Asunción: MTESS. Disponible en: <http://www.trabajo.gov.ar/left/estadisticas/ conlab/informesa.asp $>$. Acceso en: 24 feb. 2018.

MURILLO, Maria Victoria. 2005. Sindicalismo, coaliciones partidarias y reformas de mercado en América Latina. Buenos Aires: Siglo XXI.

ORGANIZACIÓN INTERNACIONAL DEL TRABAJO. 2016. Panorama Laboral America Latina y el Caribe 2016. Lima: Oficina Regional OIT para America Latina y el Caribe.

POULANTZAS, Nicos. 1980. Estado, poder y socialismo. Madrid: SigloXXI.

WAINER, Andrés; GAGGERO, Alejandro; SCHORR, Martin. 2014.

Restricción eterna: El poder económico durante el kirchnerismo. Buenos Aires: Futuro Anterior.

ZAPATA, Francisco. 1993. Autonomía y subordinación en el sindicalismo Latinoamericano. México: Fondo de Cultura Económica. . 2003. ¿Crisis en el sindicalismo en América Latina? Kellog Institute Working Paper, n. 302, pp. 1-26. 


\title{
VOLVIENDO A LAS BASES: EL SINDICALISMO ARGENTINO FRENTE A LOS RETOS DEL CAPITALISMO ACTUAL
}

\author{
BRUNO DOBRUSIN
}

JUAN MONTES CATO

Resumen: El sindicalismo en la fase actual del desarrollo capitalista argentino enfrenta enormes desafíos relacionados con el avance de la formulación de políticas neoliberales que se expresan en la profundización de la flexibilidad, el crecimiento de los mercados informales, la retirada del Estado como actor central en las relaciones laborales y el auge de las empresas multinacionales en tanto agentes que operan a escala global. Estos procesos interrogan las formas de resistencia y confrontación que marcaron un gran período de conflictividad. En gran parte esa dinámica estuvo regimentada por el modelo corporativista que articuló las relaciones laborales. En este artículo nos interesa analizar las implicancias de ese modelo, señalar en qué medida se viene desmontando y cómo esta transformación reenvía el accionar sindical a los lugares de trabajo, es decir a la confrontación con el capital en las instancias de producción.

Palabras clave: Sindicalismo; Corporativismo; Conflicto Multinacionales.

\section{REORGANIZING FROM THE BELOW: CONTEMPORARY CAPITALISIM AND THE CHALLENGES FOR ARGENTINE TRADE UNIONISM}

Abstract: In the current stage of capitalism, the Argentine labour movement is facing significant challenges due to the advance of neoliberal policies that deepen flexibilization, informality and enlarge the power of large corporations in relation to the State. These processes put into question the forms of resistance and confrontation in a period of increasing labour-capital tensions. This article 
analyzes the consequences of neoliberal policies over the traditional corporatist model of labour relations in Argentina. It looks at how this transformation is reorganizing trade union action and the need to return to workplace-based unionism as a way to present a direct challenge to capital.

Keywords: Trade Unionism; Corporatism; Conflict; Multinationals.

Recebido: 12/06/2018 Aprovado: 28/06/2018 